\title{
Part I: Historical Research
}




\title{
ORIENTAL STAR MAPS
}

\author{
F. Richard Stephenson \\ Department of Physics. \\ University of Durham. \\ Durham, DH1 3LE, U.K.
}

\begin{abstract}
The development of celestial mapping in China is discussed with particular regard both to the accuracy of construction and present-day utility of star maps from the pre-Jesuit period.
\end{abstract}

\section{INTRODUCTION}

Apart from its own intrinsic interest, the study of the history of oriental celestial cartography has proved of considerable value in modern astronomy. Perhaps the most important aspects are the investigation of the past orbit of Halley's Comet and identification of the remnants of historical supernovae. Commencing around $200 \mathrm{BC}$. numerous astronomical observations were reported in the history of China. Later - towards the end of the first millennium AD Japan and Korea followed suit. Regular observations in all three countries continued until recent centuries. The positions of comets and stellar outbursts (supernovae and novae) and, where appropriate, their movements are often described in considerable detail relative to nearby star groups. Such records have been systematically investigated in recent years (e.g. Kiang, 1971: Clark and Stephenson. 1977: Stephenson and Yau, 1985). The value of Far Eastern observations in such researches is emphasised by the dearth of useful data from other parts of the world before the European Renaissance.

In this paper the principal characteristics of ancient and medieval oriental star maps will be discussed, with some comments on the gradual development of celestial mapping. Points of special concern will be: (i) the accuracy of positioning of the stars displayed on surviving charts illustrated by selecting easily recognisable bright stars and ( $i$ ) the identification of the constituents of the various oriental asterisms in terms of their western equivalents. Both Japan and Korea adopted the traditional astronomy of China but there appears to be little in the way of original star maps from either of these countries (Needham, 1959). After about AD 1600, traditional East 
Asian celestial cartography was considerably diluted by the influence of Jesuit astronomers. some of whom attalned high positions in the Chinese court. Hence this investigation will concentrate on the period prior to AD 1600.

Throughout this paper the terms "orlental". "East Asian" and "Far Eastern" wlll be reqarded as synonomous. All refer to China together with its cultural satellites Japan and Korea. The Wade-Giles system of romanisation is adopted for the names of Chinese dynasties, places - with the exception of the capital Beijing - and star groups.

\section{CHARACTERISTICS OF ORIENTAL STAR MAPS}

Fig 1 shows a fairly typical oriental star map. Although this is a rather late example (dating from AD 1453), it has much in common with charts produced in earlier times. The original chart is painted on the ceiling of Lung-fu temple in Beijing. An excellent drawing - copied here - is published by Academia Sinica (1978).

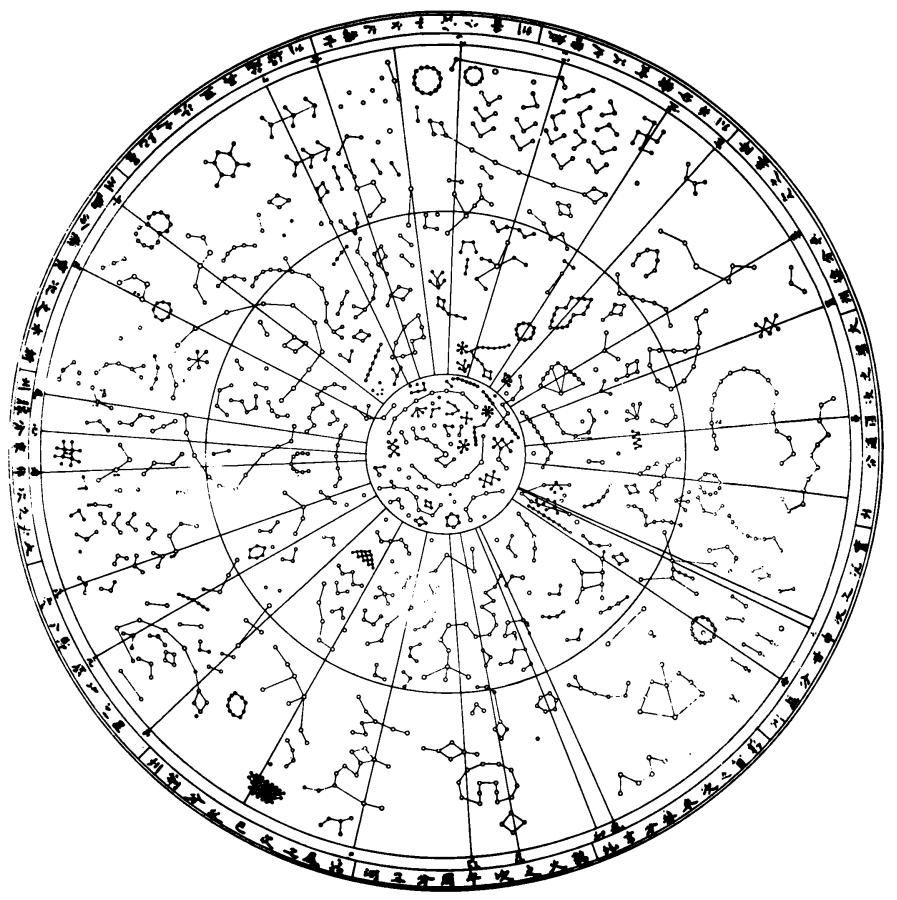

Fig 1. A typical oriental star map 
Some 1500 stars are represented, divided into roughly 300 groups. This and similar works exhibit several features not usually found on early Western star maps. Perhaps the most obvious of these is the complete lack of idealised figures such as animals, ancient deities, etc: instead, there is an evident concern for mapping the visible sky. The motive for celestial observation was very much astrological: the night sky was regarded as the counterpart of the Chinese Empire. Hence asterisms were named after the emperor and his family, courtiers, government officials, etc. as well as buildings and other everyday objects (Ho Peng Yoke, 1966). However, it seems clear that the various star groups were regarded as only nominally representing their terrestrial equivalents: their actual shape was of relatively minor importance.

East Asian star maps are decidely equatorial, unlike early charts from the occidental world which until relatively modern times preferred an ecliptical framework. Fig 1, which is on a polar projection, portrays the night sky along with a fairly complex co-ordinate system. Moving outwards from the centre, the three concentric circles are respectively the circle of perpetual visibility, the celestial equator and the circle of perpetual invisibility at the edge of the chart. The declinations of the inner and outer circles are close to + and -60 deg, more suitable for south China than the latitude of Beijing ( $+40 \mathrm{deg})$. The visible sky is divided into 28 unequal zones radiating from the north celestial pole. These are the hsiu ("lunar mansions"), named after 28 star groups lying in the general region of the celestial equator. The hsiu were numbered eastward from Chueh ("Horn") in Virgo. From ancient times, it was customary to measure the R.A. of stars, comets, etc. in tu (degrees, numbering 365.25 to a circle) relative to one of these zones. Declination was expressed in terms of angular distance from the north celestial pole.

Perhaps the most regative aspect of oriental celestial cartography is the lack of concern for the relative brightness of the stars. Virtually all extant maps make. no distinction between stars of different brightness. The explanation may be astrological; provided a star was recognised as belonging to some asterism, its influerice within that group was regarded as independent of its brightness. This lack of discrimination makes identification of stars relative to their western equivalents much more difficult than would otherwise have been the case.

Although the number of stars on a typical far Eastern chart is not too dissimilar from that catalogued in the Almagest (i.e. 1022 stars), only 48 constellations were recognised by Ptolemy. Hence Far Eastern asterisms tend to be much smaller than those so familiar to western astronomy. The number of stars in a group is seldom more than 10 while 
many asterisms contain only a single star. The fact that very few oriental asterisms resemble the occidental constellations is merely an indication of the random arrangement of the stars which are visible to the unaided eye. Among the notable exceptions to this rule are pei-tou ("The Northern Dipper"), identical with the Plough in Ursa Major and Shen ("The Triad"), equivalent to the main portion of Orion.

The 28 lunar mansions are 1 llustrated in Fig 2. This diagram is a copy of a carving on a tombstone at Han-chiang in Chiang-su province dating from around AD 900 (Astronoma Sinica, 1978). Although these hsiu lie roughly on a great circle, this is nelther identical with the celestial equator nor the ecliptic.

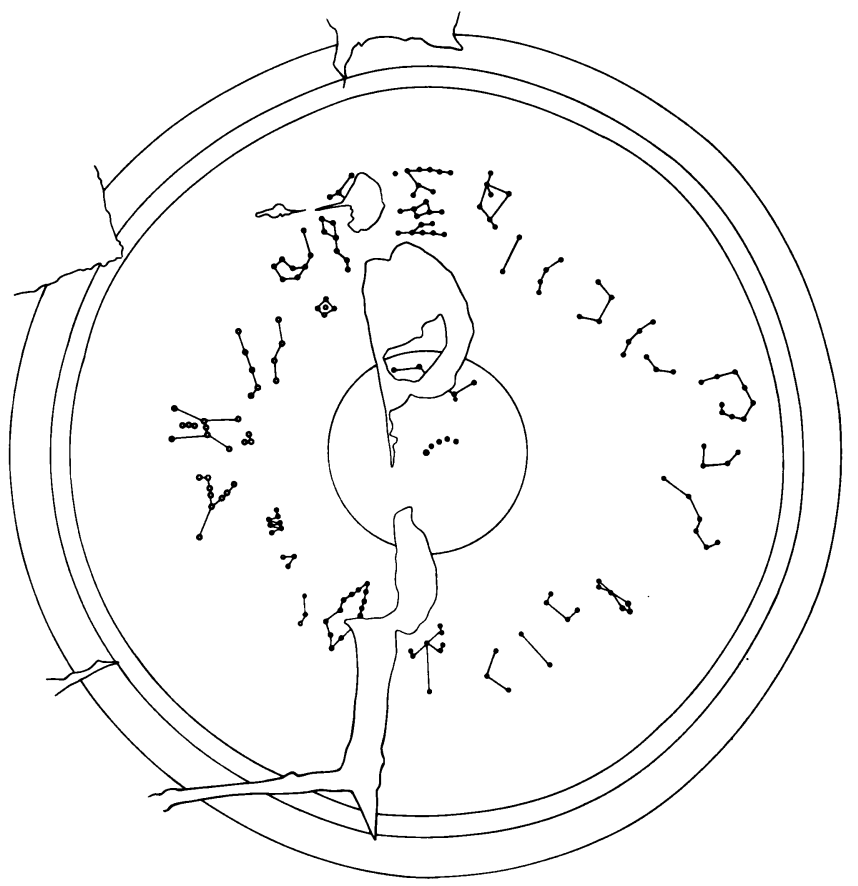

Fig 2. Stars of the 28. lunar mansions 
Applying precession, it is apparent that in the remote past the mean circle through these asterisms approximated better to the celestial equator than at present. This has led to much speculation on the origin of the hsiu, most recently by Kistemaker and Yang (1987 - paper in the present volume). In each case an epoch of origin in the 3rd millennium BC is deduced. At the present time, documentary evidence is not helpful. There are no extant historical records of any sort from China before about $1500 \mathrm{BC}$. Even well into the 1 st millennium $B C$, only the names of a few lunar mansions are preserved. The earliest surviving list of all 28 hisiu dates from 433 BC. This is inscribed on a wooden box and was found in 1978 while excavating a tomb in Ho-pei province (Wang Jianmin, 1979).

\section{DEVELOPMENT OF STAR MAPPING IN CHINA}

The oldest extant star maps from China which have any claim to accuracy are fairly late (soon after AD 1000). However, Chinese history gives evidence of reasonably careful celestial mapping some 1500 years earlier. The astronomical treatise of the Chin-shu ("History of the Chin Dynasty"), compiled around $\mathrm{AD} 635$, implies that detailed star charts were constructed in the 4 th century $B C$, notably by the great astronomer Shih Shen (Ho Peng Yoke, 1966). This was during the Warring states Period, more than a century before the unification of China into a single empire (221 BC). These charts are said to have contained a total of 1464 stars in 283 groups - figures which were to become almost canonical in later times. Sadly these charts were lost around the time the Chin-shu was composed.

An incomplete copy of an ancient star catalogue survives in late texts ( 8 th century AD). This work, the Hsing-ching ("Star Manual") gives the equivalent R.A. and dec. of the about 120 stars. Positions are typically specified to the nearest half degree. Although tradition assigns this catalogue to Shih Shen (c $350 \mathrm{BC}$ ), a detailed study of the individual measurements by Maeyama (1977) indicates a date some 3 centuries later: $70 \pm 30 \mathrm{BC}$. Despite the lack of extant star maps from this early period. it is clear from the accuracy of the measurements preserved in the Hsing-ching that fully 2,000 years ago the means was available to construct a fairly precise chart.

What may be a survival of ancient astrography is found in two distinct artefacts. These are (i) a 14 th century Korean star map engraved on stone and (ii) a bronze planisphere of uncertain date and origin recovered last century from a Japanese junk. The former, which dates from $A D$ 1395, is in Chang-duk Palace, Seoul and was extensively studied by Rufus (1913) and Rufus and Chao (1944). The 
latter is housed in the Royal Scottish Museum. Edinburgh, and has been examined by Knobel (1909) and the author (Clark and stephenson, 1977). Each chart is on a polar projection, extending to a declination of approximately - 55 deg, and follows the general pattern of Fig 1 . However, the ecliptic - incorrectly depicted as a circle - is shown in addition to the usual equatorial co-ordinate system. The Milky Way ( $T$ 'ien-ho. "the Celestial River") is prominently featured.

A count by Rufus (1913) on the korean map indicated 1463 stars (virtually the conventional figure) grouped in 306 asterisms. The two charts reveal almost identical astrography and it is clear that both share a common origin. The map from the Japanese junk is devold of any inscription and its origin is thas obscure. However, the text which accompanies the Korean map states that it is based on an ancient rubbing of a previous stone chart which had been lost during a battle in $A D 672$. At some unknown period. perhaps centuries before, the original chart had been presented by a chinese emperor to one of the three kingdoms into which Korea was divided. From the position of the equinoxes recorded in the Korean inscription ("in the east. a little preceding the 5 th degree of Chueh and in the west a little beyond the 14 th degree of $K^{\prime}$ uei"), a date of $30 \pm 10$ $B C$ can be calculated. This is only a few decades after the computed date of the Hsing-ching catalogue as derived by Maeyama (1977). A similar date $1 \mathrm{~s}$ indicated from measurements on both the Korean and Japanese maps (locations of the equinoxes and Polaris).

In order to check the positional accuracy with which the stars are represented on both of these charts. I have identified the positions of 20 of the brightest stars as listed in a modern catalogue. Correcting for precession to the epoch $30 \mathrm{BC}$, the measured declinations of these stars have a standard error as large as 5 deg. However, as will be demonstrated below, only one extant pre-Jesuit map (from around AD 1200) achieves significantly better precision than this. It is particularly interesting to note that several asterisms are represented quite differently from those on medieval Chinese maps.

Although Chinese histories tell of several star maps and globes produced by leading astronomers at various times during the first millennium $A D$, only crude artefacts survive from this long interval. We have to proceed to the sung Dynasty (AD 960 - 1279) to find preserved star maps which show evidence of careful construction. Only two charts are deserving of comment here but both are well known to historians of astronomy. The earlier of these is the oldest printed star map from any part of the world (Needham et al.. 1960). Divided into five sections, it is included in a treatise composed between $A D 1088$ and 1094 by the great astronomer Su Sung. An excellent illustration is provided 
by Academia Sinica (1978). Three of these sections are drawn using a polar projection and show respectively (i) the north circumpolar region. (ii) the northern hemisphere and (iii) the southern hemisphere down to -60 deg declination. This last section shows the sky in the south polar region as a void. (It was not until the time of the Jesuit astronomers that oriental star maps began to illustrate the region of sky not visible from China). The other two maps are on a plate carree projection and respectively depict the stars between (iv) 12 and 24 hours R.A. and ( $v$ ) 0 and 12 hours R.A. On the various sections, the circle of perpetual visibility, celestial equator, ecliptic and lunar mansions are represented. The total number of stars represented is stated to be 1456, divided into 283 asterisms: these are virtually the ancient canonical numbers.

Despite their association with su sung, all five charts are surprisingly crudely produced. Many asterisms have an idealised form and an appreciable number of roughly circular and linear asterisms can be discerned. Analysis of the declinations of the same 20 bright stars as discussed above shows that the standard error in this co-ordinate is high, some 4 deg. Such a map, although giving the impression of skilful preparation, could not have been of great astronomical value.

A much more precise map is the su-chou (Soochow) chart, formerly located in a temple at su-chou in Chiang-su province and now housed in a Su-chou museum. This work was extensively studied by Rufus and $T^{\prime}$ ien (1945). It was prepared in $A D 1193$ for the instruction of the heir to the Chinese throne and was engraved on stone roughly half a century later. Academia Sinica (1978) have published an excellent rubbing. The su-chou chart depicts the sky visible from central China (roughly 35 deg north latitude) on a polar projection. The customary equatorial co-ordinate system is shown together with the ecliptic (incorrectly represented as circular). The Milky Way is also displayed. Although the inscription accompanying the chart states that there are 283 asterisms and 1565 stars (essentially the conventional figures), a count by Rufus and $T^{\prime}$ ien (1945) revealed as many as 313 asterisms but only 1440 stars. Certain brilliant stars - e.g. Sirius (Lang - "the Celestial Wolf") and Canopus (Lao-ien, "the Longevity star") are indicated by larger symbols than usual but there is no systematic attempt to distinguish between stars of different brightness.

Measurement of the declinations of the sample 20 bright stars reveals a tolerably small standard error of about 1 deg. This was clearly a well produced map, an example of the high standard of astronomical achievment reached during the sung Dynasty.

No star charts are preserved from the Yuan Dynasty

$(A D$ 
1279 - 1368) which followed the Sung, although this was also a period of considerable astronomical activity. The official history of the period, the Yuan-shih ("History of the Yuan Dynasty") lists a wide variety of instruments used in the imperial observatory at Beijing. Amorig these was a celestial globe "made to give a miniature representation of the heavens". However, when this and a similar globe were examined by Jesuit astronomers around AD 1600, any star markings had already been worn away by weathering (Needham. 1959).

Several detailed star maps - such as the Lung-fu map shown in Fig 1 - have survived from the subsequent Ming Dynasty (AD $1368-1644$ ). However, no extant artefact of pre-Jesuit origin is executed with much regard to accuracy. The standard error in declination of bright stars displayed on the Lung-fu chart proves to be as high as 7 deg. obviously a painting on the ceiling of a tomb may not necessarily represent the best of Ming celestial cartography. However, other charts of this period - e.g. a well preserved stone engraving at Chang-shu in Chiang-su province dating from AD 1506 - are similarly imprecise. Based on the available evidence, we can only confirm the impression of astronomical decadence in Ming China as recounted by the Jesuits (Needham, 1959).

\section{IDENTIFICATION OF ORIENTAL ASTERISMS}

Some of the difficulties which are encountered when attempting to identify the constituents of the 300 or so oriental star groups have already been mentioned in passing. obviously, since only about 25 or 30 per cent of all naked-eye stars are displayed on a typical chart. most visible stars must never have been grouped into asterisms. Probably all stars brighter than about magnitude +4 are sufficiently prominent to be recognised as belonging to a particular group but whether fainter stars were included or overlooked must have depended very much on local configurations. Such speculations inevitably lead to queries on the stability of form of many of the falnter asterisms over two millennia. The use by orlental astronomers of crudely drawn star maps which made little if any distinction between stars of radically differing brightness would not improve this situation.

The identification of oriental asterisms in relation to Western star catalogues has attracted attention since Jesuit astronomers such as Ferdinand Verbiest investigated the problem in the 17 th century. In recent years several charts have been produced which plot the positions of naked-eye stars and overlay these with the probable outlines of the Chinese asterisms. Among these works might be mentioned 
those of Ho Peng Yoke (1962 and 1966) and Yi Shitong (1982). Such maps serve a wide range of purposes but their value is somewhat limited since they tend to concentrate on the data in charts and catalogues produced by the Jesuit astronomers. What has yet to be produced is a similar map based on the stars as represented in a pre-Jesuit planisphere.

As noted in section 3 , the Korean and Japanese star maps probably illustrate Chinese celestial cartography of the $1 \mathrm{st}$ century $B C$ and according to Maeyama (1977) the data in the Hsing-ching catalogue appears to be of similar date. More than a thousand years later - mean epoch AD 1150 - both the Su-chou and Su Sung charts are valuable representations of medieval Chinese astrography. These works are supported by the Huang-yu star catalogue dating from a century earlier (Pan Nai and Wang De-chang, 1981). Finally, several rather crude Ming star maps of pre-Jesuit construction are available from around $A D$ 1500. Herice at least in principle it should be possible by comparing with modern catalogues to produce similar maps to those of Ho Peng Yoke and Yi Shitong for three distinct periods of Chinese history. In the author's opinion, such an undertaking would be invaluable. It would greatly assist the study of the development of celestial cartography in China and would be of considerable importance in cometary and supernova studies. An investigation of this sort for the Cassiopeia region alone was made by the author (Stephenson, 1971: - see also Clark and stephenson, 1977). The motive here was a search for the remriant of a likely supernova observed in this area by Chinese and Japanese astronomers in AD 1181. However, as far I am aware, no other region of sky has been studied in similar detail.

The asterisms in the ecliptical zone offer much scope for this type of investigation, as was recognised by Liu Ci-yuan (1980). From around 200 BC until recent centuries. the astronomical treatises of the various Chinese dynastic histories record numerous conjunctions of the Moon and planets with asterisms. A typical example is of the following form: "Ying-huo (Mars) trespassed against the 2 nd star from the north of Fang (an asterism in scorpius)". The exact date is almost invariably given, which enables the precise lunar or planetary position to be computed. Using this detailed information, it should be possible to accurately map most of the zodiacal asterisms at various periods in Chinese history.

\section{REFERENCES}

Academia Sinica, 1978. An Album of Anclent Relics and Documents Connected With Astronomy. (Ed. Institute of Archaeology). Wenwu Chubanshe, Beijing. 
Clark, D.H. and and Stephenson, F.R., 1977. The Historical Supernovae. Pergamon Press, Oxford.

Ho Peng Yoke, 1962. 'Ancient and medieval observations of comets and novae in Chinese sources'. Vistas in Astronomy. 5, 127-225.

Ho Peng Yoke, 1966. The Astronomical Chapters of the Chin Shu. Mouton, Paris.

Kiang, T. 1971. 'The past orbit of Halley's Comet'. Mem. Roy. Astr. Soc., 76, 27-66.

Kistemaker, J. and Yang Zhengzong, 1987. 'A new approach to traditional Chinese astronomy' - see this volume.

Knobel, E.B., 1909. 'On a Chinese planisphere'. Mon. Not. Roy. Astr. SoC. 69, 435-443.

Liu Ci-yuan, 1986. 'The Chinese names of fifty zodiacal stars in the 4th-6th century'. Acta Astronomica Sinica. 27. $276-278$.

Maeyama, Y., 1977. 'The oldest star catalogue of China. Shih Shen's Hsing Ching'. In Prismata (ed. Y. Maeyama and W.G. Saltzer), Franz Steiner Verlag, wiesbaden.

Needham. J. 1959. Science and Civilisation in China. vol. 3. Cambridge Univ. Press, Cambridge.

Needham, J., Wang Ling and Price, D. J. de S., 1960. Heavenly Clockwork. Cambridge Univ. Press, Cambridge. 1960 .

Pan Nai and Wang De-chang, 1981. 'The Huang-you star of the Song Dynasty - a Chinese star list of the early medieval period'. Chinese Astron. Astrophys., 5, 441-448.

Rufus, W.C. 1913. 'The celestial planisphere of King Y1 Tai-jo'. Trans. Korea Br, Roy, Asiatic Soc., 4, 23-72.

Rufus, W.C. and Chao, C. 1944. 'A Korean star map'. Isis, $35,316-326$.

Rufus, W.C. and T'ien Hsing-chih, 1945. The Soochow Astronomical Chart. Univ. of Michigan Press. Ann Arbor.

Stephenson, F.R., 1971. 'A suspected supernova in AD 1181". l. J1. Roy. Astr. Soc., 12, 10-38.

Stephenson, F.R. and Yau, K.K.C., 1985. 'Far Eastern 
observations of Halley's Comet: $240 \mathrm{BC}$ to AD $1378^{\circ}$. J. Brit, Interplan, Soc.. 38, 195-216.

Yi Shitong, 1982. All-Sky Star Maps. Ditu Chubanshe, Beijing.

Wang Jianmin, 1979. 'On a vessel inscribed with the 28 lunar lodges, the Green Dragon and the white Tiger, found in the tomb of Zeng Houyi'. Wenwu, No 7, 40-45. 
Discussion:

WAYMAN Is dating of early maps by the equinoxes supported by the position of the pole star? STEPHENSON Yes, but whereas accuracy from equinoxes dates the map even to 10 years, the pole star only gives 50 years, but it is important to verify that the whole star map reflects the epoch, not just the equinoxes. 